\title{
Different biological effectiveness of ionising and non-ionising radiations in mammalian cells
}

\author{
E. Schmid ${ }^{1}$ and T. Schrader ${ }^{2}$ \\ ${ }^{1}$ University of Munich, Radiobiological Institute, 80336 Munich, Germany \\ ${ }^{2}$ Physikalisch-Technische Bundesanstalt (PTB), 38116 Braunschweig, Germany
}

\begin{abstract}
It has been discussed ever since among the scientific communities, whether non-ionising radiation can alter or harm biological systems. While the mechanisms of underlying effects of ionising radiation are well understood and described here, there is less understanding in the effects caused by non-ionising radiation except for heating effects. Recent results have been interpreted, that numerical chromosome aberrations may be induced by exposure to RF radiation. Our own experiments employing the $\mu$ TEM cell yield results similar to findings observed for aneugenic-inducing chemical agents which can cause spindle disturbances. For comparison purpose to the effects caused by those agents we used the well established human-hamster hybrid $\left(\mathrm{A}_{L}\right)$ cell line to evaluate the biological effects after exposure to RF radiation $(835 \mathrm{MHz})$.
\end{abstract}

\section{Ionising radiation}

Ionising radiation (IR) can randomly cause damage to all cellular components and induces a variety of DNA lesions, like single strand breaks, double strand breaks or base damage. The deposition of energy from IR results in the formation of excited and ionized molecules. Traditionally, IR damage to DNA is ascribed to both direct and indirect effects. Direct effects result from the direct interaction of the IR with DNA. Indirect effects result from the interaction of DNA with reactive species formed by IR. The underlying chemistry of lesion formation is fairly well understood. The resulting biological effect on mammalian cells is influenced by the dose, dose-rate, and quality of the radiation. The reason is that the biological effectiveness depends on the spatial distribution of the energy imparted and the density of ionisations per unit path length of the ionising particles. The energy loss per unit

Correspondence to: T. Schrader

(thorsten.schrader@ptb.de) path length of such a charged particle, the so-called "linear energy transfer" (LET) is responsible for the determination of the relative biological effectiveness (RBE), which equals the ratio of the absorbed doses of two types of IR that produce the same biological effect. It is well documented that high-LET radiation induces energy-dependently higher RBE values for various biological endpoints than low-LET radiation. However, it has also been recognised during the last decades that, especially at low doses, low-LET radiations do not all have the same effectiveness. These findings are not surprising, because already the wide range of LET values covered by the photon and electron radiations is suggesting considerable RBE differences.

The most reliable and detailed data on the RBE of lowLET and high-LET radiations exist for a well quantifiable IR effect in human cells, i.e. the production of dicentric chromosomes in lymphocytes of peripheral blood samples irradiated in vitro. For example, such a radiation-induced dicentric chromosome in a peripheral blood cell is given in Fig. 1. These structural chromosome alterations are the most thoroughly studied biological sentinel of radiation-induced cellular injury and are considered to be a reliable biological indicator of human radiation exposure. However, it should be taken into account that dicentric chromosomes are not radiation-specific, i.e. an exposure to ionizing radiation cannot necessarily be deduced from an elevated aberration rate. Nonetheless, they can be viewed as characteristic of radiation. The basis for a cytogenetic dose estimation are doseresponse curves (calibration curves) for IR-induced dicentric chromosomes. For this reason, whole blood is irradiated in vitro with different IR qualities at different doses. After culture of lymphocytes and preparation of chromosomes, the chromosome analysis is done by light microscopy. The induction of comparable yields of dicentric chromosomes in lymphocytes, irrespective of their exposure in the body or in vitro, is a main prerequisite for the suitable application of these calibration curves to estimate an individual's exposure.

Published by Copernicus Publications on behalf of the URSI Landesausschuss in der Bundesrepublik Deutschland e.V. 


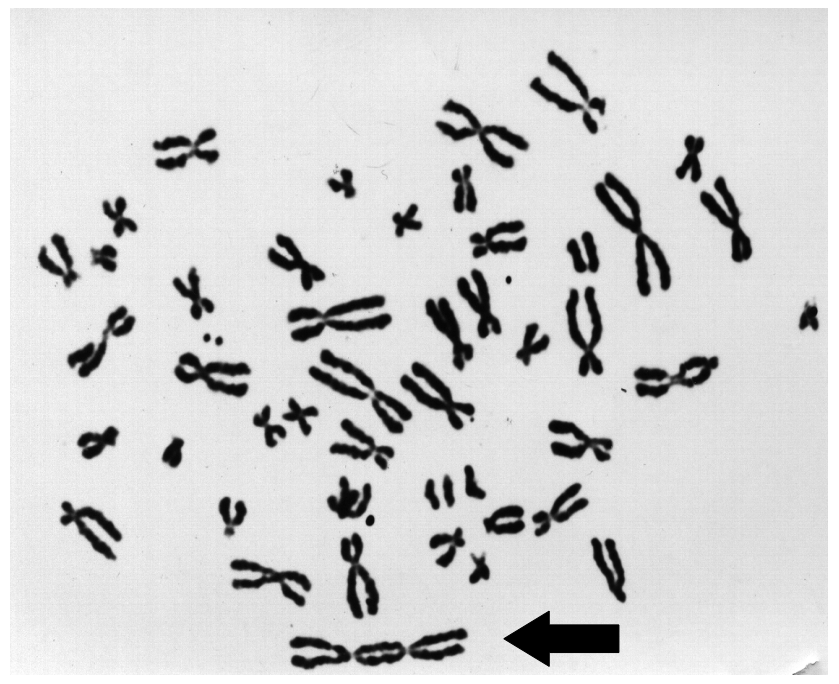

Fig. 1. Human peripheral lymphocyte showing a radiation-induced dicentric chromosome (arrow).

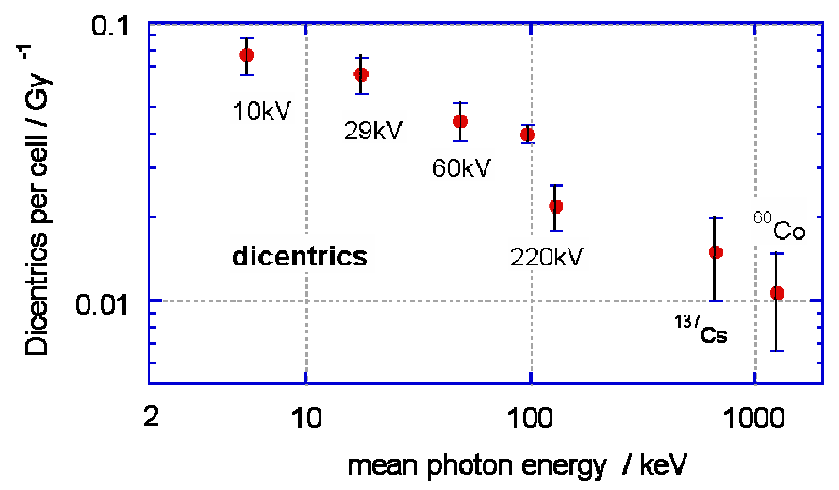

Fig. 2. The linear coefficients $\alpha$ (and standard errors) of the linearquadratic dose-response $\left(\mathrm{y}=\alpha \mathrm{D}+\beta \mathrm{D}^{2}\right)$ for the induction of dicentric chromosomes by different photon energies in human lymphocytes from the same donor. The data were determined in our laboratory and demonstrated by the BEIR VII report (BEIR, 2006).

This has been proven for whole-body irradiation in animal experiments with different species and could be confirmed for therapeutic whole-body exposure of cancer patients with ${ }^{60}$ Co $\gamma$-rays (Schmid, et al., 1974).

In vitro dose-response curves for dicentric chromosomes, established with appropriate fitting procedures, are now available for most IR qualities relevant for radiation protection purposes. In particular, our group obtained extensive data sets for the induction of dicentric chromosomes in human lymphocytes by different IR qualities (Krumrey et al., 2004; Schmid et al., 2003). In part, the low dose effectiveness (linear coefficients of linear-quadratic dose-response curves) of these data sets have been listed by the International Commission on Radiological Protection, ICRP (2003), and the National Research Council, NRC, of USA (BEIR, 2006).
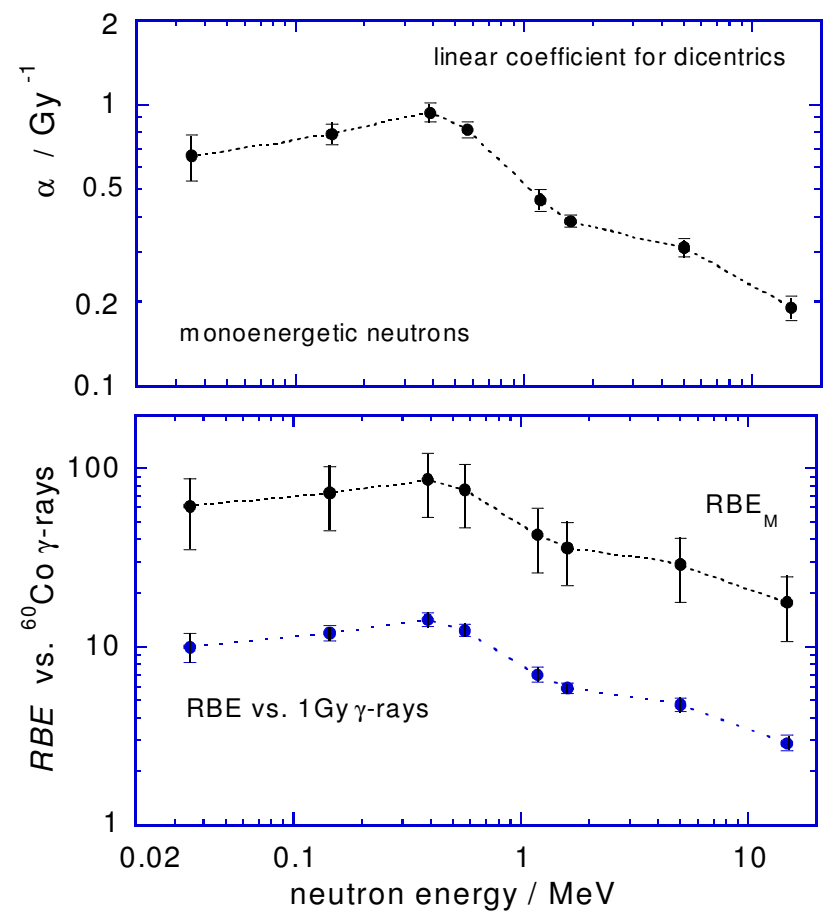

Fig. 3. Upper panel: The linear coefficient $\alpha$ (and standard error) from a linear-quadratic fit to the dose dependence for dicentric chromosomes induced in human lymphocytes by mono-energetic neutrons of different energies. Lower panel: The relative biological effectiveness at minimal doses $\left(\operatorname{RBE}_{M}\right.$, and standard errors) versus ${ }^{60} \mathrm{Co} \gamma$-rays. The curve below gives the high-dose RBE versus 1 Gy of ${ }^{60} \mathrm{Co} \gamma$-rays. The data were determined in our laboratory and demonstrated by the ICRP (2003).

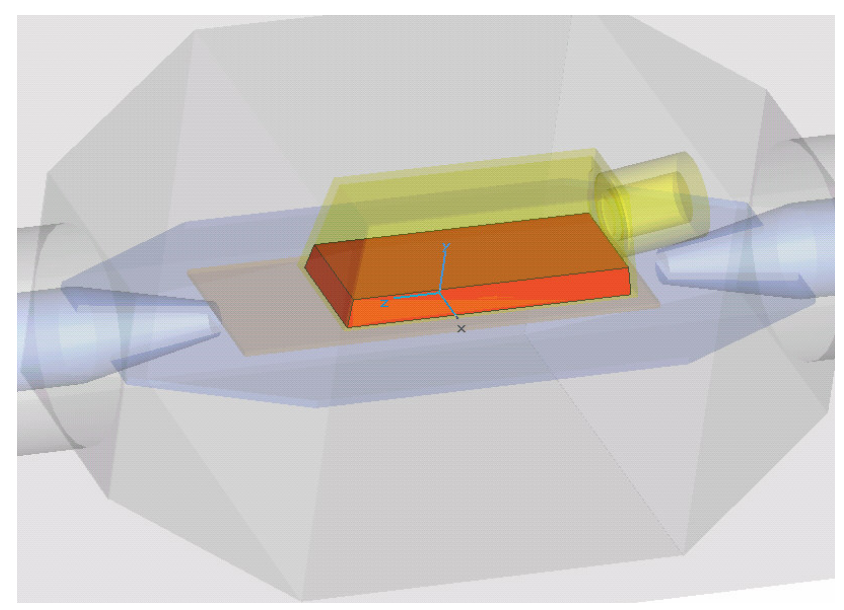

Fig. 4. 3D-model of the so-called $\mu$ TEM cell for exposure of the biological samples to RF radiation under far-field conditions (flask with monolayer of $\mathrm{A}_{L}$ cells plus $5 \mathrm{ml}$ medium on the $\mu \mathrm{TEM}$ cell septum) used for numerical evaluation and dosimetry.

Examples for photons and neutrons are given in Figs. 2 and 3 , respectively. 


\section{Non-ionising radiation}

In contrast to our knowledge on the underlying mechanisms of IR-induced biological effects, the experimental data regarding biological effects of chronic exposure to nonionising radiation (NIR) at typical frequencies of GSM mobile phones (900 and $1800 \mathrm{MHz}$ ) are fragmentary and inconsistent in many respects. Since an understanding of the biophysical mechanisms for the observed biological effects should help to rationalise the data, some understanding of the underlying mechanisms is needed. In principle, there are two ways by which biological effects as a result of exposure to radiofrequency (RF) radiation can occur. These are by thermal (heating) effects or as a result of possible non-thermal effects. The energy quanta of the NIR quality at 900 and $1800 \mathrm{MHz}$ equal 4 and $7 \mu \mathrm{eV}$, respectively (IEGMP, 2000). Both these values are extremely small compared with the energy of typically around $1 \mathrm{eV}$ needed to break the weakest chemical bond in a DNA molecule. However, in contrast to this experience, the primary focus of a large number of in vivo and especially in vitro investigations during the last years was to evaluate the ability of RF radiation to induce DNA damage in rodents, cultured rodent and human cells as well as freshly collected human lymphocytes using DNA alterations, structural chromosome aberrations or micronuclei. The results of these studies are conflicting, although there is a general tendency to believe that these biological effects are rather unlikely. An expert review panel (Moulder et al., 2005) concluded that the majority of the in vitro studies reporting a positive effect were flawed due to poor biological design and/or inadequate dosimetry. Therefore, it is essential to perform further investigations under well-defined experimental conditions and scoring criteria in order to resolve the controversy concerning the possible risks associated with $\mathrm{RF}$ radiation in the human body, especially at the cellular level. Recent results obtained in mammalian cells after exposure to RF radiation have been interpreted as the production of numerical chromosome aberrations (Mashevich et al., 2003; Tice et al., 2002). Such a mechanism would be different to that induced by ionising radiation but could be similar to findings observed for aneugenic-inducing chemical agents which can cause spindle disturbances in cells resulting in numerical chromosome aberrations.

Therefore, our aim was to evaluate the predicted aneugenic capacity of RF radiation as emitted from GSM mobile phones, applying a fast and useful test for detecting possible spindle disturbances in single cells which has been used in our laboratory during the last 30 years. Analysis of spindle disturbances were carried out in an established humanhamster hybrid $\left(\mathrm{A}_{L}\right)$ cell line after exposure to RF radiation $(835 \mathrm{MHz})$. For the microwave exposure of the biological samples $\left(\mathrm{A}_{L}\right.$ cells with medium) a special setup of the PTB was used. This so called $\mu$ TEM cell is demonstrated in Fig. 4. The applied $\mu$ TEM cell was developed and manufactured 1994 for the exposure of biological systems at the Insti-

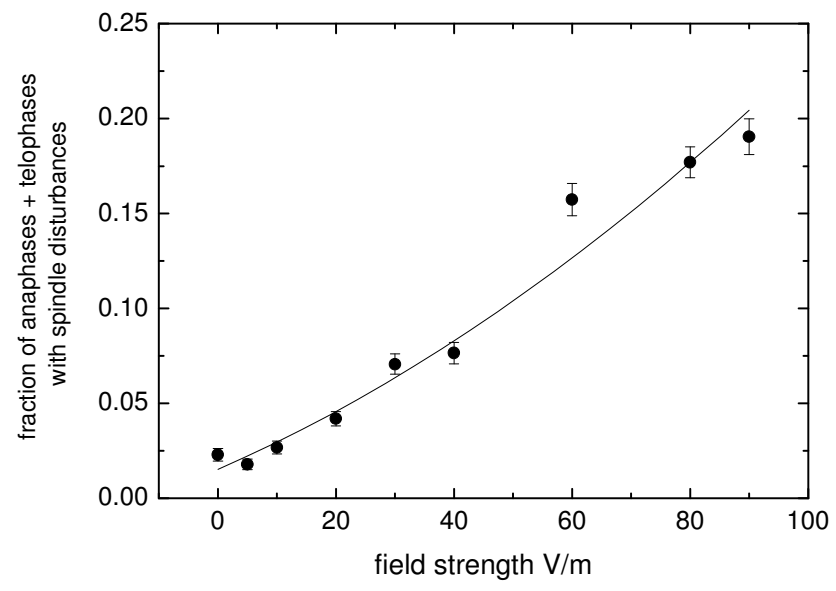

Fig. 5. The fraction of the damaged anaphases plus telophases (stages of the mitotic cycle) versus the applied electrical field strengths from $5 \mathrm{~V} / \mathrm{m}$ to $90 \mathrm{~V} / \mathrm{m}$.

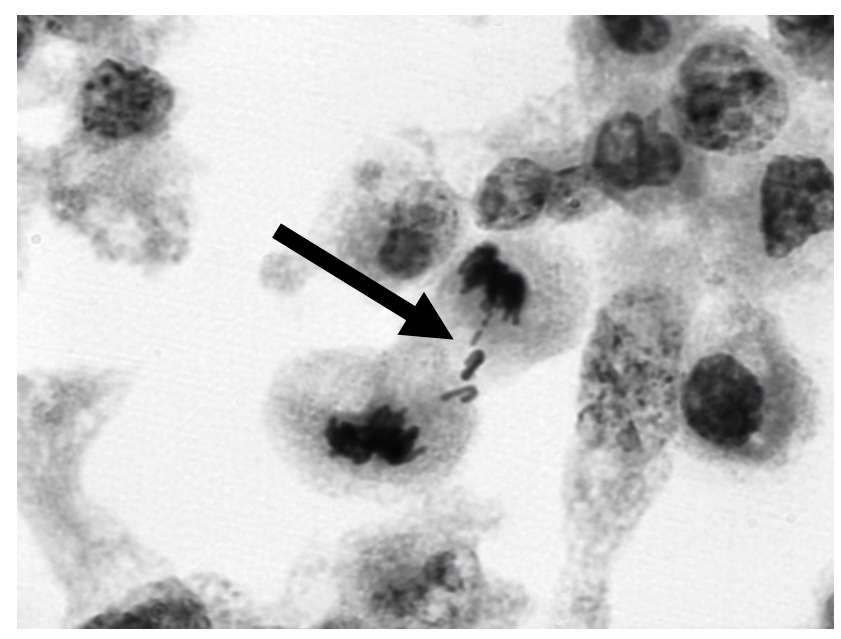

Fig. 6. Anaphase stage of the mitotic cycle of $\mathrm{A}_{L}$ cell with spindle disturbances (lagging of two chromosomes) induced by RF radiation (arrow).

tute for Communications Technology at Braunschweig Technical University (Germany). Its design rules follow the concept of the Crawford (1974) cell, but it is optimized in terms of the Voltage-Standing-Wave-Ratio (VSWR). For the frequency $(835 \mathrm{MHz}$ ) used in our investigation, ICNIRP (1998) limits the electric field strength to $86.7 \mathrm{~V} / \mathrm{m}$ and $39.7 \mathrm{~V} / \mathrm{m}$, respectively. Therefore, the influence of electric field strengths from $5 \mathrm{~V} / \mathrm{m}$ to $90 \mathrm{~V} / \mathrm{m}$ on the $A_{L}$ cells was investigated at a constant exposure time of $0.5 \mathrm{~h}$. With increasing field strength there is an obvious tendency for an increase in the fraction of the damaged anaphases and telophases, i.e. in the induction of spindle disturbances. The fraction of the damaged anaphases plus telophases (stages of the mitotic cycle) versus the applied field strengths from $5 \mathrm{~V} / \mathrm{m}$ to $90 \mathrm{~V} / \mathrm{m}$ is given in Fig. 5. The line is only inserted to improve the read- 
ability of the graphs and, therefore, is without mathematical meaning. The reason is that up to now no reasonable explanations are available which may help to elucidate the underlying mechanisms involved in RF radiation-induced spindle disturbances. In Fig. 6, there is an anaphase stage of the mitotic cycle with spindle disturbances (lagging of two chromosomes) observed after exposure to RF radiation (continuouswave, $90 \mathrm{~V} / \mathrm{m}, 0,5 \mathrm{~h})$.

\section{Conclusions}

Due to our experiments there is scientific evidence which suggests that there may be biological effects occurring at exposures to RF radiation, but they are certainly different to those known from IR exposure. However, in contrast to our knowledge on the IR-induced DNA alterations, the result obtained for RF radiation does not necessarily mean that these effects, i.e. spindle disturbances, lead to disease or injury, but it is a potentially important information for evaluating the underlying mechanisms.

\section{References}

Crawford, M. L.: Generation of Standard EM Fields Using TEM Transmission Cells, IEEE Trans. EMC, EMC-16, 189-195, 1974.

ICNIRP Guidelines: Guidelines for limiting exposure to timevarying electric, magnetic, and electromagnetic fields (up to 300 GHz), Health Phys., 74, 494-522, 1998.

ICRP: Relative biological effectiveness (RBE), quality factor $(Q)$, and radiation weighting factor $\left(w_{R}\right)$, edited by: Valentin, J., ICRP Publication, 92, Elsevier Inc. New York, USA, 2003.
IEGMP: Independent Expert Group on Mobile Phone, IEGMP, Mobile phones and health, NRPB, UK, http://www.iegmp.org.uk, 2000 .

NRC: Health risks from exposure to low levels of ionizing radiation, BEIR VII, Phase 2, The National Academies Press, Washington, D.C., USA, 2006.

Krumrey, M., Ulm, G., and Schmid, E.: Dicentric chromosomes in monolayers of human lymphocytes produced by monochromatized synchrotron radiation with photon energies from $1.83 \mathrm{keV}$ to $17.4 \mathrm{keV}$, Radiat. Environ. Biophys., 43, 1-6, 2004.

Mashevich, M., Folkman, D., Kesar, A., Barbul, A., Korenstein, R., Jerbi, E., and Avivi, L.: Exposure of human peripheral blood lymphocytes to electromagnetic fields associated with cellular phones leads to chromosomal instability, Bioelectromagn., 24, 82-90, 2003.

Moulder, J. E., Foster, K. R., Erdreich, L. S., and McNamee, J. P.: Mobile phones, mobile phone base stations and cancer: a review, Int. J. Radiat. Biol., 81, 189-203, 2005.

Schmid, E., Bauchinger, M., Bunde, E., Ferbert, H. F., and von Lieven, H.: Comparison of the chromosome damage and its dose-response after medical whole-body exposure to ${ }^{60}$ Co $\gamma$ rays and irradiation of blood in vitro, Int. J. Radiat. Biol., 26, 31-37, 1974.

Schmid, E., Schlegel, D., Guldbakke, S., Kapsch, R. P., and Regulla, D.: RBE of nearly monoenergetic neutrons at energies of $36 \mathrm{keV}-14.6 \mathrm{MeV}$ for induction of dicentrics in human lymphocytes, Radiat. Environ. Biophys., 42, 87-94, 2003.

Tice, R. R., Hook, G. G., Donner, M., McRee, D. I., and Guy, A. W.: Genotoxicity of radiofrequency signals. I.Investigation of DNA damage and micronuclei induction in cultured human blood lymphocytes, Bioelectromagn., 23, 113-126, 2002. 